\title{
LE ROLE NUTRITIONNEL CHEZ LA VACHE EN LACTATION DES ACIDES ACÉTIQUE ET BUTYRIQUE FORMÉS AU COURS DE L'ENSILAGE (suite) (1)
}

PAR

\section{Z. ZELTER}

Laboratoire de Recherches de la chaire de Zootechnie Institut National Agronomique, Paris

\author{
DEUXIÈME PARTIE
}

\section{MODALITES TECHNIQUES ADOPTEES POUR NOS RECHERCHES}

\author{
CHAPITRE I
}

\section{METHODES EXPERIMENTALES APPLICABLES A LA VACHE EN LACTATION}

La réalisation correcte d'une étude de nutrition avec vaches laitières se heurte à un bon nombre d'obstacles matériels.

Ia période de stabulation, pendant laquelle il est possible de mesurer avec précision les ingesta individuels, s'échelonne sous nos climats de novembre à avril. La nécessité d'une longue préparation des sujets pour l'expérience, réduit la durée effective des observations à une centaine de jours seulement.

L'interférence d'un grand nombre de facteurs exogènes et endogènes est susceptible de masquer les effets de la nutrition étudiée sur les activités mammaires. Pour écarter ces influences, un cheptel bovin important, permettant d'opérer un choix approprié de sujets d'expérience, est indispensable. Actuellement, seules des exploitations privées possèdent chez nous de tels effectifs, que les éleveurs hésitent à exposer aux aléas d'une expérience risquant de compromettre momentanément la productivité des animaux.

(1) Voir Annales de Zootechnie, $\cdot 1953, n^{\circ}$ r. 


\section{A. - Difficultés du choix des sujets d'expérience}

L'élimination des influences qu'exercent l'évolution physiologique de la lactation et la gestation, limite le choix des animaux d'expérience à ceux dont l'activité mammaire aura dépassé le maximum au moment où débute l'essai et n'excèdera pas son $7^{\mathrm{e}}$ mois à la fin de celui-ci.

L'élimination des interventions dues aux facteurs de race, d'individualité et d'état de nutrition, n'est possible, et encore dans une certaine mesure seulement, que dans des troupeaux sélectionnés et bien tenus, dont les antécédents du sujet peuvent être suffisamment connus du chercheur pour permettre la constitution de groupes d'animaux comparables.

L'effectif de ces troupeaux comprend couramment une quarantaine de vaches, dont une vingtaine vêlent au moment de l'entrée en stabulation. Il n'est guère possible, dans ces conditions, de disposer pour l'expérience de plus de Io à I5 sujets à la fois.

\section{B. - Techniques expérimentales}

Le chercheur qui entreprend une expérience sur des vaches en lactation n'a à sa disposition que deux techniques, dont aucune n'est exempte de critiques. Celles-ci se prêtent cependant bien à une étude de nutrition et conduisent à des résultats satisfaisants $(34,48)$.

\section{Io Technique de "périodes successives "}

Un nombre restreint d'animaux ne permet pas la constitution de groupes comparables. La technique de "périodes successives " mise au point par KELLNER et KUHN, est la seule possible dans ce cas.

La méthode est basée sur le fait que la décroissance de l'activité mammaire chez la vache ne s'effectue pas par bonds successifs, mais revêt normalement une allure régulière dans un laps de temps relativement court. On admet, en conséquence, que, dans une expérience de brève durée, la chute de lactation est la même, ou tout au moins parallèle, au début et à la fin de celle-ci, si les conditions d'entretien et de nutrition restent inchangées.

La technique consiste à soumettre un groupe de sujets à un régime alimentaire de référence, pendant des périodes de pré et de post-expérience. Dans l'intervalle de ces deux périodes, on intercale une ou plusieurs périodes expérimentales, durant lesquelles le régime étudié est substitué à celui de référence.

Les mesures des sécrétions mammaires effectuées au cours des pré et post-expériences servent à calculer, au moyen d'une régression linéaire, la chute moyenne journalière de lactation due à son évolution physio- 
logique naturelle. On admet que la décroissance aurait été la même pendant les périodes intercalaires, si le régime alimentaire n'avait pas été modifié entre temps, et on calcule par intrapolation les productions théoriques que, dans ce cas, il aurait été possible d'observer.

L'écart constaté entre les données théoriques ainsi calculées et celles réellement observées au cours des périodes expérimentales, est supposé traduire l'effet produit par l'emploi du régime soumis à l'épreuve.

HENKE et GOO (28) ont appliqué cette mtéthode lors de leurs études de l'influence des niveaux variés de protéines sur la sécrétion lactée. MorRIs (49) l'a employée dans l'étude comparée de la valeur biologique des protéines alimentaires pour la production laitière.

Nous avons eu recours fréquemment à cette technique, que nous avons légèrement modifiée en effectuant le calcul de la décroissance journalière de la lactation d'après une régression exponentielle, et non pas linéaire, la première représentant d'une façon plus précise la loi de la persistance des sécrétions mammaires chez la vache (4I).

L'absence de groupe de contrôle constitue évidemment un des plus sérieux reproches que l'on puisse faire à cette technique. Celle-ci présente cependant l'avantage de permettre l'observation du comportement d'un même animal dans des conditions de nutrition variées.

\section{$2^{\circ}$ Techniques de groupes " comparables "}

La méthode mise au point par FJORD a été utilisée en premier par les stations danoises de recherches.

Le principe consiste dans la formation d'au moins deux groupes de sujets comparables par la race, le nombre de parturitions, l'état de lactation et de gestation et par le niveau des sécrétions mammaires. Les observations faites lors d'une période de préexpérience pendant laquelle tous les sujets sont soumis à un régime de référence donné, permettent la constitution de lots identiques de vaches. Ceci fait, on institue une période expérimentale durant laquelle l'un des groupes sert de témoin.en restant au régime de référence, tandis que l'autre subit le régime expérimental.

Il est préférable, lorsque les conditions le permettent, d'achever l'essai par une post-expérience, pendant laquelle le dernier groupe est remis au régime témoin.

La comparaison des écarts de production observés entre les deux groupes de vaches durant la période d'expérience avec ceux existant dans la pré et la post-expérience, sert de critère de jugement de l'influence exercée par le changement de régime.

Cette méthode est plus précise que celle de périodes successives. Son application implique cependant la nécessité de pouvoir disposer de 
nombreux animaux pour former des groupes parfaitement comparables, ce qui n'a pas toujours été notre cas.

I,es circonstances - fort variables avec les troupeaux sur lesquels il nous a été donné d'entreprendre nos recherches - nous ont empêché de nous tenir à une seule technique expérimentale et nous ont amené à adopter tantôt l'une, tantôt l'autre des deux méthodes que nous venons d'exposer.

\section{CHAPITRE II}

\section{NORMES ALIMENTAIRES DE LA VACHE LAITIËRE}

Nous avons vu dans la première partie de ce travail que l'épanouissement complet des fonctions mammaires se trouve en partie seulement sous la dépendance de l'hérédité et de l'individualité ; le milieu, et plus particulièrement le niveau de nutrition, exercent sur cet épanouissement une action prépondérante.

La glande mammaire de la vache se montre, en effet, singulièrement sensible aux apports de principes calorigènes et de protides. I,es carences minérales et vitaminiques paraissent moins affecter l'intensité des sécrétions mammaires.

$I_{t}$ a mise au point d'un niveau de nutrition individuel apte à favoriser au maximum la capacité productive de la glande, nécessite chez la vache une longue période de tâtonnements, qu'un recours à des normes alimentaires appropriées permet d'abréger. Pour le nutritionniste, celles-ci représentent un outil de travail conventionnel il est vrai, mais fort commode. L'établissement d'un rationnement optimum doit néanmoins tenir compte d'un fait bien prouvé : chez un sujet à cycle génital normal, une alimentation excessive ne peut empêcher l'involution naturelle et progressive de l'activité fonctionnelle de la mamelle.

Nous nous devons, bien entendu, de justifier les normes alimentaires que nous avons appliquées à nos animaux d'expérience. Étant donné leurs fondements classiques, nous nous contenterons de rappeler seulement les notions les plus caractéristiques dont ils procèdent.

\section{A. - Normes énergétiques}

Il n'est pas possible de discuter ici les théories énergétiques en présence. Signalons cependant que, dans le domaine de la nutrition des animaux domestiques, les divergences portent essentiellement sur les modes d'évaluation et d'expression de l'énergie des besoins, et des principes nutritifs. Les normes elles-mêmes, quelle que soit la théorie sur laquelle elles sont basées, ne présentent entre elles que des écarts absolus insignifiants. 


\section{Io Modes d'évaluation de l'énergie disponible préconisés en nutrition animale}

On sait qu'en nutrition humaine et d'animaux de laboratoire, la détermination de l'énergie physiologiquement utile des principes calorigènes digérés, s'effectue à l'aide des coefficients de conversion calorifique de ATwatTER : 4, 9, 4. Ces coefficients sont cependant inapplicables au ruminant, car ils ne tiennent pas compte des pertes dues à la digestion microbienne qui est très importante chez cette espèce animale.

En effet, les processus fermentaires auxquels sont exposés dans le rumen les principes calorigènes ingérés par le polygastrique, donnent lieu à la formation d'une énorme masse gazeuse qui se traduit par une perte d'énergie évaluée à 60 calories environ par Ioo g de glucides digestibles ingérés $(36,2)$. Dans le cas de la vache en lactation, la perte quotidienne globale d'énergie peut atteindre 4 ooo calories (II).

La plupart des auteurs reconnaissent, par conséquent, la nécessité de déduire de l'énergie contenue dans la fraction digestible des principes nutritifs, celle perdue par le jeu des activités bactériennes, afin d'aboutir à l'énergie physiologiquement disponible, appelée encore énergie métabolisable, chez le ruminant. Celle-ci représente, selon Armsiy (2), la somme de l'énergie nette et de la chaleur excrétée au cours du processus nutritif (A. D. S.).

Or, Brody (8) considère que, si chez le carnivore, l'action dynamique spécifique est due principalement à la chaleur libérée dans le foie par l'excès d'acides aminés, chez le polygastrique la digestion bactérienne a l'inconvénient d'occasionner en outre un " heat increment " qui résulte de l'ingestion du repas. L'animal ne possédant qu'un très faible pouvoir de contrôle sur cette énergie libérée, celle-ci constitue une perte supplémentaire ajoutable à 1'A. D. S.

Ceci fait, que les principes nutritifs ingérés par le ruminant perdent beaucoup de leur efficacité énergétique, et rend compréhensible l'existence, en matière de nutrition d'animaux domestiques, de deux doctrines différentes pour l'évaluation de l'énergie, doctrines sur lesquelles reposent les normes modernes d'alimentation rationnelle de ces animaux.

\section{a) DOCTRINE DE L'ÉNERGIE MÉTAABOLISABLE}

Ce sont surtout les nutritionnistes américains qui se recommandent de cette doctrine, basée sur le principe suivant : dans des conditions optima de nutrition, les coefficients de mutations dynamiques dans l'organisme sont invariables, quels que soient les éléments calorigènes. Ils 
admettent, par conséquent, que l'énergie métabolisable fournit une base d'évaluation satisfaisante de l'énergie disponible.

Dans le cas du ruminant, cette énergie métabolisable se détermine en appliquant à la somme des principes digestibles totaux, calculés d'après la formule (glucides digestibles + protides digestibles + lipides digestibles $\times 2,25$ ), le coefficient de conversion calorifique 3,6 (r7).

Dans cette méthode, l'unité nutritive adoptée est la 'T. D. N. (Total Digestible Nutrients), qui équivaut à une livre anglaise de principes digestibles totaux ( $453 \mathrm{~g}$ ), et qui exprime d'une façon indirecte l'énergie métabolisable. Un kg de T. D. N. correspond par conséquent à 3600 calories métabolisables chez le ruminant.

\section{b) DoctrRine de L'ÉNERGIE NETT'TE}

KeLLNER (36) arguant que le coefficient de mutation de l'énergie métabolisable en énergie nette diffère avec la nature des principes calorigènes, affirme que le premier mode d'évaluation énergétique donne une idée très imparfaite de l'énergie réellement convertie par l'organisme pour son entretien ou ses productions. D'où la notion " d'énergie nette » formulée par cet auteur et qui constituerait, selon lui, le seul critère d'estimation énergétique acceptable chez l'animal domestique. Ce point de vue est partagé par Hansen, Armsby, Moligaard, Craseman, Leroy et d'autres encore.

La conception d'énergie nette est représentee par un certain nombre d'étalons de mesure actuellement utilisés dans l'alimentation animale, dont nous rappelons les équivalences énergétiques. "I'unité amidon " de KELLNER correspond à 2360 calories d'énergie nette. Le "Therm " préconisé par ARMSBY équivaut à I ooo calories nettes. "1'Équivalent fourrager » de HANSEN, adopté en France par LEROY, a une valeur énergétique nette de I 850 calories chez le rúminant. Convertie en énergie métabolisable, cette unité contient $27 \mathrm{I} 7$ calories.

Reconnaissons cependant le caractère purement conventionnel et le manque de rigueur scientifique de tous ces équivalents nutritifs, dont l'efficacité énergétique réelle est sous la dépendance de l'espèce animale, de la nature et du niveau de production du sujet. Les faits observés prouvent toutefois que, dans leur ensemble, les normes énergétiques modernes prouvent toutefois que, dans leur ensemble, les normes énergétiques modernes permettent de calculer avec une approximation suffisante les besoins de l'animal et la couverture de ces besoins.

\section{$2^{\circ}$ Besoins énergétiques de la vache en lactation}

Les travaux fondamentaux de KELLNER (36), de JORDAN (32), de Armsby (2), de Forbes (I9), de Forbes et Kriss (I8), signalent une 
grande variabilité individuelle dans le taux d'utilisation de l'énergie alimentaire chez la vache en lactation. Smith et RICE (57) croient d'ailleurs à l'existence d'une corrélation entre l'intensité de la production et le coefficient d'utilisation énergétique, qui serait héréditaire et se retrouverait chez le même sujet pendant ses lactations successives.

Ceci montre, par conséquent, que les normes alimentaires qui sont des ordres de grandeurs valables surtout pour des groupes d'animaux, nécessitent des ajustements appropriés pour permettre la satisfaction des besoins individuels d'entretien et de production de la vache en lactation.

\section{a) BESOINS D'ENTRETIEN}

La couverture théorique des besoins du métabolisme basal est calculée, selon la " loi de surfaces " de RUBNER, qui se trouve liée au poids corporel $\mathrm{du}$ sujet par la relation $\mathrm{S}=\mathrm{K} \mathrm{P}^{x}$, qui est une expression de MEEH (I6) midifiée.

En fait, la vie d'un sujet en stabulation entraîne une dépense énergétique accrue (2). Les travaux classiques de BEACH, de KELI,NER, de FINGERLING et de ARMSBy ont montré, en outre, que la nature du régime alimentaire et le travail de consommation et d'assimilation de ce régime augmentent également les dépenses métaboliques de l'entretien.

Les normes que nous rapportons ci-contre et qui nous ont servi pour le calcul des besoins d'entretien de nos sujets d'expérience prévoient des majorations, destinées à couvrir les dépenses énergétiques supplémentaires occasionnées par ces divers facteurs.

\section{Normes d'entretien pour vaches en lactation}

\begin{tabular}{|c|c|c|c|c|}
\hline $\begin{array}{c}\text { Poids vif } \\
\text { (du sujet en } \mathrm{kg} \text { ) }\end{array}$ & $\begin{array}{c}\text { Unités } \\
\text { fourragères } \\
\text { (d'après LERoy) }\end{array}$ & $\begin{array}{c}\text { U. F. converties } \\
\text { en énergie } \\
\text { métabolisable } \\
\text { par l'égalité } \\
\text { I UF }=2717 \text { cal. }\end{array}$ & $\begin{array}{l}\text { T. D. N. en kg } \\
\text { (d'après BRoDY) }\end{array}$ & $\begin{array}{l}\text { T. D. N. } \\
\text { converties en } \\
\text { énergie métabolique } \\
\text { par le coef. de con- } \\
\text { version calorifique } \\
3,6\end{array}$ \\
\hline 400 & 3,5 & $9.5^{10}$ & 2,80 & 10.080 \\
\hline 500 & 4,0 & Io. 868 & 3,27 & II. $77^{2}$ \\
\hline 600 & 4,5 & I 2.226 & 3,77 & 13.572 \\
\hline 700 & 5,0 & I 3.585 & 4,23 & 15.228 \\
\hline 800 & 5,4 & I 4.672 & 4,63 & 16.668 \\
\hline
\end{tabular}

La comparaison de deux séries de données citées fait constater qu'entre les prévisions énergétiques de LEROY, exprimées en énergie nette, et celles de Brody exprimées en T. D. N. c'est-à-dire en énergie métabolisable, 1'écart dépasse rarement xo \%. 


\section{b) BESOINS DE PRODUCTION}

La synthèse propre des constituants du lait n'exigerait pas de dépense notable d'énergie $(5,54)$, tandis que 1'activité musculaire de la glande mammaire durant la traite en occasionnerait une, mais légère (49).

Les besoins de production de la vache se trouvent par conséquent principalement liés à l'énergie contenue dans le lait sécrété. Cette énergie est fonction de la richesse lipidique de la sécrétion $(25,22,28)$. GaINEs (22) la calcule sur la base d'un lait standard à $4 \%$ de lipides, dont la valeur énergétique par $\mathrm{kg}$ est estimée à 750 calories. L'équation utilisée à cet effet par l'auteur est la suivante : $Q=0,4 M+15 F(Q=$ quantité de lait à $4 \% ; \mathrm{M}=$ quantité de lait sécrété $; \mathrm{F}=$ richesse lipidique du lait).

Ia moyenne de normes de production qui résulte des travaux de divers auteurs américains tels que Gaines, Brody et Procter, Forbes et KRISS, est de $0,305 \mathrm{~kg}$ TDN par $\mathrm{kg}$ de lait à $4 \%$ de graisse, ce qui correspond à I 098 calories d'énergie métabolisable. En France, LEROY estime que, chez la vache en lactation, les besoins énergétiques de production diffèrent avec le niveau de celle-ci, et recommande une norme moyenne de 0,38 unités fourragères, également par $\mathrm{kg}$ de lait à $4 \%$ de matière grasse, soit donc environ I 030 calories d'énergie métabolisable.

Pratiquement, les besoins de production de nos animaux d'expérience ont été couverts à raison de I 098 calories, afin de procurer à ceuxci un léger excès d'énergie, destinée à servir de volant de sécurité.

\section{B. - Normes protidiques}

La glande mammaire semble manifester une sensibilité plus prononcée pour les carences azotées qu'énergétiques. En effet, un régime énergétiquement équilibré, mais déficient en protides, entraîne chez la vache une chute très rapide des sécrétions de lait et de matière grasse.

L'apport protidique pose à la fois un problème quantitatif et qualitatif.

\section{$1^{\circ}$ Aspect qualitatif du besoin protidique}

La recherche d'un niveau minimum de protéines, compatible avec une productivité maxima de la glande mammaire, procède essentiellement de considérations d'ordre économique.

Des nombreux travaux de recherche (IO, 29) confirment la grande variabilité du taux de l'utilisation azotée chez la vache en lactation signalée antérieurement par JORdan, KEILINER et MOLLGaARd. Selon HALNAN (24), les meilleurs coefficients d'utilisation observés dans la pratique sont de l'ordre de $70 \%$. 
Selon Buschmann (Io), le besoin protidique de la vache en lactation se trouve satisfait par un rapport :

$$
\frac{\text { protéines digestibles du régime }}{\text { protéines contenues dans le lait }}=\mathrm{I}, 4
$$

HENkE et GoO (28) observent que des régimes alimentaires renfermant I0,8\% et $20,2 \%$ de protéines digestibles procurent des productions lactées sensiblement analogues.

Nous affirmerons, en accord avec MORRIs (49), qu'un accroissement du taux de protides ingérés contribue à élever jusqu'à un certain niveau seulement les sécrétions mammaires, qui tendent par contre à diminuer si l'optimum de l'apport en question se trouve dépassé.

\section{$2^{\circ}$ Aspect qualitatif du besoin protidique}

On admet que la majeure partie du besoin protidique global de l'organisme doit être satisfaite par de l'azote différencié, constitué par des acides aminés indispensables. Une légère part de ce besoin peut cependant être couverte par de l'azote de forme banale, non protéique (asparagine, urée, etc...) (58).

L'efficacité de 1'apport azoté est donc fonction au premier chef de l'équilibre des aminoacides qu'il renferme. Or, on sait que le ruminant est incapable d'effectuer la synthèse du tryptophane et de la lysine qui sont indispensables à son entretien et à sa croissance $(5 \mathrm{I}, 47)$. Il est également prouvé que l'absence de ces deux acides aminés, et plus particulièrement de lysine, dans les protides du régime alimentaire de la vache en lactation, constitue un facteur limitant de la sécrétion mammaire (49).

Les aliments usuels de la vache sont rarement déficients en cystine ou en hystidine. Certains d'entre ces aliments se rapprochent davantage du lait par leur contenu en aminoacides, et conviennent par conséquent mieux pour l'activité physiologique de la mamelle (42). Ainsi, les fourrages seraient plus riches en tryptophane que les farines animales; les pois, les haricots, les tourteaux de graines oléagineuses, l'orge et l'avoine sont plus aptes à assurer une bonne sécrétion lactée que le maîs et le blé $(26)$.

On considère généralement qu'une ration alimentaire comportant un mélange de plusieurs aliments bien choisis, apporte à la vache des quantités suffisantes d'acides aminés nécessaires pour l'entretien et la lactation.

Jusqu'ici, un certain désaccord paraît exister au sujet de l'utilisation de l'azote aminé pour la satisfaction des exigences protidiques de la vache. Des expériences relativement récentes prouveraient que l'azote 
non différencié est susceptible d'assurer la couverture partielle des besoins du ruminant soumis à un régime protéique déficient $(90,38)$.

Certains auteurs (62) affirment que le polygastrique synthétise des protéines corporelles à partir d'urée. Frens (20), BARTLETT et BLAXTER (4) considèrent que l'azote non protéique ne présente qu'un très faible intérêt pour la production laitière, tandis que OWEN, SMITT et WRIGHT (53), ainsi que HASTINGS (27), pensent au contraire que la vache en lactation en tire un réel profit, tout au moins pour ses besoins d'entretien. Delage et François (I2) partagent cette dernière opinion.

De l'avis de FERrando (I5), l'observance de la qualité de l'apport protéique importe moins chez le polygastrique que chez le monogastrique.

On sait que la valeur biologique des protides végétaux est moindre que celle de protides animaux. Les aliments consommés par la vache renferment, en outre, sensiblement moins de lysine que le lait. Ceci a conduit la plupart des auteurs à recommander, par mesure de sécurité, une forte majoration du taux des protéines alimentaires ingéré par cet animal. L'application du coefficient 2 proposé par SAVAGE (56) et admis également par LEROY (42), est jugé trop élevé par MORRISON (50).

Nous nous résumerons donc en précisant que les normes azotées d'entretien recommandées pour la vache sont de $0,5 \mathrm{~g}$ de matières protéiques digestibles par $\mathrm{kg}$ de poids vif en France, contre $0,6 \mathrm{~g}$ figurant couramment dans les tables d'alimentation anglo-saxonnes.

Les besoins azotés de production préconisés actuellement diffèrent avec les auteurs. Ainsi, FrEDERIKSEN recommande $60 \mathrm{~g}$ de protéines digestibles par $\mathrm{kg}$ de lait à $4 \%$ de graisse; KELLNER et FINGERLING évaluent ces mêmes besoins à $62 \mathrm{~g}$, Mollganard à $55 \mathrm{~g}$, Armsby à $49 \mathrm{~g}$, CRASEMAN à $50 \mathrm{~g}$ et I EROY à $60 \mathrm{~g}$. C'est ce dernier chiffre que nous avons adopté, car nous avons admis, lors du calcul du contenu azoté du régime alimentaire de nos vaches, l'utilisation de la fraction non protéique présente en quantités appréciables dans les fourrages et les racines. L'équivalent protéique de ces aliments a été évalué d'après la formule inđiquée par KAY (33) :

Equivalent protéique $=\frac{\text { Mat. azot. totales digest. }+ \text { mat. prot. digest. }}{2}$.

\section{C. - Normes minérales}

Les besoins de la vache en éléments minéraux sont incomplètement connus pour le moment.

La plupart des auteurs insistent sur le fait que la balance du calcium reste toujours négative chez les fortes laitières, pendant la première partie de la lactation, en dépit de la présence dans le régime alimentaire d'un 
taux calcique très élevé. Ceci montre, par conséquent, que les possibilités d'absorption de cet élément chez la vache sont limitées. Il est toutefois admis que les effets d'une insuffisance calcique se manifestent au niveau de la glande mammaire, seulement lorsque l'animal a perdu au moins $20 \%$ de ses réserves.

Une carence phosphorée provoquerait par contre une chute plus rapide des sécrétions mammaires.

Selon BREIREm (7), qui rapporte les observations faites récemment par Slagsvord, des apports très élevés ¿de phosphore et de calcium n'auraient aucun effet favorable sur la richesse du lait en matière grasse.

Pour MORris (49), les besoins en phosphore et en calcium sont liés, dans une certaine mesure, au rapport de ces deux éléments dans le régime alimentaire ingéré, rapport qui conditionne leur solubilité et leur absorption au niveau intestinal.

D’une façon générale, les fourrages de bonne qualité, en particulier le foin de luzerne, constituent une excellente source de $\mathrm{Ca}$ pour la vache. Seules les vaches recevant une trop faible quantité de ces aliments auraient besoin d'un apport supplémentaire d'éléments minéraux (I6).

Des besoins en chlorures de sodium, quoique insuffisamment précisés, sont également admis pour deux raisons : les aliments végétaux ingérés par la vache renferment des concentrations élevées en potassium; des quantités importantes de ClNa sont exportées par les sécrétions mammaires et impliquent nécessairement une compensation.

Le maintien d'un équilibre physiologique de $\mathrm{K} / \mathrm{Na}$ convenable rend par conséquent indispensable un apport supplémentaire de ClNa.

On reconnaît, en outre, l'utilité d'une incorporation supplémentaire de l'élément magnésium dans le menu quotidien de la vache, vu l'importance qui est attachée au rapport $\mathrm{Ca} / \mathrm{Mg}$.

Soulignons aussi la grande méconnaissance actuelle du rôle des oligo-éléments dans la nutrition de la vache laitière. Quoiqu'il soit certain pour l'instant que les aliments courants ingérés par la vache en contiennent, on ignore toutefois si les quantités apportées correspondent aux besoins physiologiques.

Précisons enfin que la présence, dans la ration quotidienne, de $2,2 \mathrm{~g}$ de $\mathrm{Ca}$ et de $2,2 \mathrm{~g}$ de $\mathrm{P}$ par Ioo $\mathrm{kg}$ de poids vif, suffit, d'après certains auteurs américains (I) à couvrir les besoins de la vache laitière en ces éléments. Les normes françaises prévoient des apports beaucoup plus élevés :

\begin{tabular}{|c|c|c|}
\hline $\mathrm{Ca}$ & $\mathrm{P}$ & $\mathrm{ClNa}$ \\
\hline - & - & . \\
\hline 5 & 3 & \\
\hline 4 & 3 & 2 \\
\hline
\end{tabular}




\section{D, - Besoins en vitamines.}

Le ravitaillement en substances vitaminiques ne pose pas de véritable problème chez la vache.

L.es synthèses microbiennes des facteurs du complexe $\mathrm{B}$ et de $\mathrm{K}$ qui s'effectuent dans les réservoirs digestifs du ruminant adulte, satisfont largement les besoins de cet animal en ces substances. Notons toutefois que ces vitamines sont apportées en partie par le régime habituel $(45,65)$.

Les racines apportent en outre de fortes doses de vitamine $\mathrm{C}$, qui contribuent surtout à élever le taux de ce facteur dans le lait.

Des additions de vitamines A ou de caroténoïdes (63) et de $\mathrm{D}(3 \mathrm{I}, 37)$ exercent un effet négligeable sur l'intensité des sécrétions mammaires. Ces adjonctions accroissent uniquement l'activité vitaminique du lait sécrété.

La vitamine $\mathrm{E}$ semblerait cependant nécessaire pour le maintien de la lactation et surtout, de la prolificité (I4).

Apparemment donc, les quantités de vitamines présentes dans les aliments tels que les fourrages, les racines et les céréales, ainsi que la vie en plein air, assurent généralement à la vache les doses indispensables au maintien en bon état de fonctionnement de son organisme et au remplissement de ses fonctions physiologiques. I1 n'y a par conséquent pas à se préoccuper tout particulièrement de l'apport exogène alimentaire de substances vitaminiques.

\section{CHAPITRE III}

\section{METHODES ANALYTIQUES}

\section{A. - Méthodes de détermination de l'énergie contenue dans les ingesta}

Dans une expérience précise de nutrition, il est indispensable de connaître la composition chimique des ingesta, afin de s'assurer que les rations distribuées couvrent les besoins d'énergie et de matières protéiques des sujets d'expérience, calculées selon les normes que nous venons d'exposer.

Des techniques analytiques classiques nous ont permis de déterminer la composition chimique et la valeur nutritive des aliments utilisés au cours de nos diverses expériences (voir Rapports généraux du Ve Congrès International de Zootechnie, Paris, I949, p. 7 et suiv.). Nous les résumerons très brièvement. 


\section{I ${ }^{\circ}$ Détermination des principes nutritifs calorigènes}

a) Humidité : séchage à l'étuve à IO5 $5^{\circ}$ et à la pression atmosphérique, d'une quantité déterminée de substance, jusqu'à poids constant.

b) Matières minérales: La détermination comporte une calcination au four électrique à $55^{\circ}$.

c) Matières azotées totales: Dosage du $\mathrm{N}$ total par la technique de KJEIDAHL, comportant une minéralisation en présence de catalyseur. Le taux de $\mathrm{N}$ trouvé est ensuite converti en matières azotées totales, à 1'aide des coefficients conventionnels ci-après, qui varient, selon la nature de l'aliment :

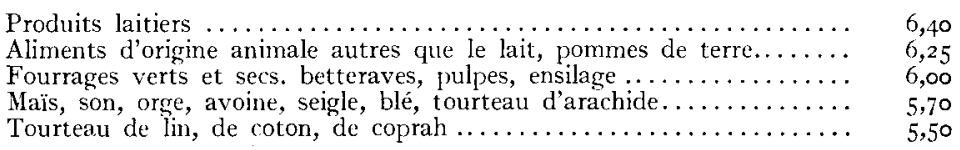

d) Matières cellulosiques : Détermination par la méthode de WEENDE, comportant successivement des hydrolyses acide et alcaline. Le résidu obtenu constitue un mélange de cellulose vraie et de lignine insoluble dans l'alcali.

e) Lipides : Le dosage comporte une double extraction continue à chaud, à l'alcool d'abord, à l'éther ensuite. Ce double épuisement facilite la libération des cénapses lipo-protéiques.

f) Extractifs non azotés: Détermination par différence selon la méthode classique qui admet (faute de technique satisfaisante de dosage direct) le calcul d'après la formule : extractifs non azotés = matière sèche - (matières azotées totales + matières grasses + matières cellulosiques + matières minérales).

\section{$2^{\circ}$ Détermination de la valeur énergétique utile des principes calorigènes}

On sait que seule la fraction digestible des principes bruts dosés chimiquement dans les aliments est convertible en énergie utile pour 1'animal. On détermine cette fraction par analogie avec celle de constituants des aliments de référence, possédant les mêmes caractéristiques d'origine et de composition chimique et figurant dans les tables de digestibilité de KELLNER. Le choix de l'aliment de comparaison des tables, de même origine, est également conditionné par les teneurs en matières cellulosiques et azotées, qui doivent être comparables.

La fraction digestible ainsi déterminée est d'abord convertie en éléments digestibles totaux (T. D. N.) d'après la formule citée au chapitre précédent. Elle est ensuite exprimée, soit en calories d'énergie métabolisable, soit en unités d'énergie nette. 


\section{$3^{\circ}$ Détermination des matières protéiques digestibles}

On les calcule à partir des matières azotées totales digestibles, à l'aide de coefficients de conversion qui figurent dans les tables de digestibilité de KELLNER pour les aliments de référence choisis.

\section{B. - Méthodes de dosage des acides gras volatils dans les ingesta}

Une étude consacrée à l'influence des acides gras volatils ingérés sur 1a lipogénèse, ne peut être réalisée sans une méthode convenable de leur estimation dans les ingesta.

Certaines techniques de dosage d'acides volatils préconisées pour les ensilages ne sont pas exemptes de critiques (43). Nous nous sommes efforcés d'employer une méthode aussi sûre et fidèle que possible.

\section{Io Méthode de Wiegner}

Rappelons que WiEgner et MAGASANik (64) se servent des courbes de distillation établies par DucLAux pour les acides volatils, afin de calculer les constantes de distillation des acides acétique et butyrique, qui sont respectivement de $36,6 \%$ et de $72,77 \%$ dans le cas d'une semidistillation. Les auteurs proposent, sur cette base, une méthode de dosage de ces acides dans les ensilages au moyen de trois semi-distillations successives, opérées à partir d'un volume constant d'extrait aqueux brut d'ensilage.

Une série d'équations établies sur la base de ces constantes de distillation, permet de calculer le taux de 1'acide acétique et de l'acide butyrique présents dans un ensilage.

GNEIST (23) fait remarquer, à la suite d'une vérification, que la méthode de WIEGNER est valable uniquement pour le dosage des acides volatils libres, mais que pour celui des acides combinés, d’autres équations sont nécessaires.

LAEPER (40) allègue de son côté que la présence d'acide lactique dans un extrait aqueux brut d'ensilage influe sur les valeurs trouvées lors des dosages séparés de l'acidité volatile libre et combinée et que, de ce fait, les équations de WIEGNER deviennent inapplicables.

D'autres auteurs $(6 I, 6)$ considèrent que certaines substances non volatiles présentes dans les extraits aqueux bruts d'ensilage, et plus particulièrement dans ceux de fourrages riches en glucides solubles ou en protéines, perturbent la marche de la distillation en exerçant, soit un effet physique sur la tension de vapeur dans les solutions, soit une action chimique à chaud, donnant naissance à des produits acides, volatils, qui faussent les valeurs trouvées. 
L'un de ces auteurs, VIRTANEN notamment (6I), essaye d'améliorer la méthode de WIEGNER tout en respectant son principe de la semi-distillation.

\section{$2^{\circ}$ Méthode de Virtanen}

Les acides volatils contenus dans l'extrait aqueux brut sont entraînés par un vif courant de vapeur d'eau. Le distillat ainsi obtenu est neutralisé, concentré et soumis à un second entraînement à la vapeur. Neutralisé à nouveau par de la baryte caustique, le $2^{\mathrm{e}}$ distillat est alors concentré et acidifié par une quantité d'acide sulfurique suffisante pour précipiter totalement la baryte. Une fraction aliquote du filtrat est alors soumise à la semi-distillation à laquelle on applique les constantes établies par WIEGNER.

Nous avons comparé les méthodes de WIEGNER et de VIRTANEN en effectuant des dosages en double sur deux ensilages de pulpes de betteraves sucrières. Les résultats de ces analyses sont rapportés ci-contre :

\begin{tabular}{|c|c|c|c|c|}
\hline & \multicolumn{2}{|c|}{ Méthode WiEgner } & \multicolumn{2}{|c|}{ Méthode Virtanen } \\
\hline & $\begin{array}{c}\text { Acide acétique } \\
\mathrm{g} \% \%\end{array}$ & $\begin{array}{c}\text { Acide butyrique } \\
\mathrm{g} \% \%\end{array}$ & $\begin{array}{c}\text { Acide acétique } \\
\text { g } \% \text { \% }\end{array}$ & $\begin{array}{c}\text { Acide butyrique } \\
\mathrm{g} \%\end{array}$ \\
\hline Pulpes $A \ldots \ldots$. & 8,60 & 0,47 & 7,65 & $\mathbf{I}, 47$ \\
\hline Pulpes $B$... & $\begin{array}{l}8,63 \\
9,65 \\
9,63\end{array}$ & $\begin{array}{l}0,46 \\
0,55 \\
0,08\end{array}$ & $\begin{array}{l}7,53 \\
9,58 \\
9,26\end{array}$ & $\begin{array}{l}1,35 \\
2,72 \\
2,53\end{array}$ \\
\hline
\end{tabular}

I,es différences sont faibles en ce qui concerne l'acide acétique. Elles deviennent très importantes pour l'acide butyrique, qui paraît être moins bien entraîné dans le dosage par la technique de WIEGNER.

I a méthode de VIR'TANEN présente cependant de nombreux inconvénients. Flle est extrêmement longue et nécessite des barbotages de longue durée et de très fortes quantités de distillat (jusqu'à 2 ooo cc). Une journée entière est nécessaire pour chaque concentration, une autre demi-jaurnée pour chaque entraînement à la vapeur et une autre demijournée encore pour l'exécution de trois semi-distillations finales.

La méthode se prête par conséquent très difficilement à des dosages en série, comme ceux que nous avions à effectuer.

\section{$3^{\circ}$ Méthode de Lepper-Flieg}

Cette méthode (39) dérive également de celle de WIEGNER, qu'elle améliore. Elle convient parfaitement aux dosages en série.

I'utilisation d'un appareillage standardisé permet d'aboutir à des constantes de distillation uniformes, ce qui n'est pas le cas de la méthode de dosage de WIEGNER. Les conditions de chauffage et d'entraînement sont très rigoureusement établies et une défécation préalable de 1'extrait 
aqueux brut évite, que des hydrates de carbone solubles soient oxydés au cours de la distillation et viennent fausser les valeurs d'acides acétique et butyrique trouvées.

Mode opératoire. - $200 \mathrm{cc}$ d'un extrait aqueux brut d'ensilage à $10 \%$, introduits dans une fiole jaugée de 250 cc sont déféqués en présence de 20 cc de lait de chaux à Io \% et de Io cc d'une solution de $\mathrm{CuSO}_{4}$ à Io \%. Laissés au repos pendant une heure, ils sont complétés ensuite jusqu'au trait de jauge et filtrés aussitôt.

$200 \mathrm{cc}$ de filtrat sont transvasés dans l'appareil de LEPPER, additionnés de $5 \mathrm{cc}$ de $\mathrm{SO}_{4} \mathrm{H}_{2}$ de densité $\mathrm{I}, 84$ en solution à $50 \%$, et soumis à une première semi-distillation de Ioo cc effectuée en 20 minutes exactement. Une deuxième semi-distillation de $50 \mathrm{cc}$ en Io minutes celle-ci, suit la première sans interruption.

Les distillats $\mathrm{D}_{1}$ et $\mathrm{D}_{2}$ ainsi obtenus sont titrés à l'aide d'une solution de $\mathrm{NaOH} \mathrm{N} / 20$.

Le calcul des teneurs en acides acétique et butyrique totaux s'effectue à 1'aide des équations établies par LEPPER et simplifiées par Fuchs (2I), dans lesquelles les valeurs $D_{1}$ et $D_{2}$ sont exprimées en cc. $\mathrm{NaOH} \mathrm{N/20}$ employés pour la neutralisation de chacun des distillats.

$$
\% \text { acide acétique total dans l'ensilage }=-0,0266 \mathrm{D}_{1}+0,1203 \mathrm{D}_{2}
$$

Selon les travaux de LepPer (39), la présence d'acide lactique dans l'extrait aqueux déféqué ne perturbe nullement les constantes de distillation des acides volatils, établies avec l'appareillage adopté.

Le dosage de l'acide lactique peut s'effectuer sur les $50 \mathrm{cc}$ de filtrat resté dans l'appareil, après la seconde distillation. On additionne ce résidu après refroidissement, de $50 \mathrm{cc}$ d'eau distillée et on soumet le tout à une attaque sulfochromique suivie d'une troisième semi-distillation de $50 \mathrm{cc}$ en Io minutes exactement. On neutralise le distillat $\mathrm{D}_{3}$ également par $\mathrm{NaOH} \mathrm{N/20.} \mathrm{On} \mathrm{calcule} \mathrm{la} \mathrm{teneur} \mathrm{en} \mathrm{acide} \mathrm{lactique} \mathrm{d'après} \mathrm{l'équation} \mathrm{:}$

$$
\% \text { acide lactique total }=-0,0082 \mathrm{D}_{1}-0,0578 \mathrm{D}_{2}+0,145 \mathrm{D}_{3} \text {. }
$$

Nous avons porté notre choix sur cette méthode, à la suite d'une vérification détaillée qui nous a prouvé sa justesse.

\section{$4^{\circ}$ Vérification de l'appareillage et de la méthode de Lepper-Flieg}

a) V'́RIFICATION A L'AIDE DE SOLUTTIONS N/2O D'ACIDES ACÉTIQUE ET BUTYRIQUE PURS, LN MÉLANGE

Des solutions $\mathrm{N} / 20$ d'acides acétique et butyrique, que nous avons préparées séparément, ont été ensuite mélangées en proportions variables. Vingt dosages successifs ont été effectués sur chaque mélange. 
$\mathrm{I}^{\circ}$ Mélange de $20 \%$ d'acide acétique et $80 \%$ d'acide butyrique en solution $\mathrm{N} / 20$.

Prise d'essai : Ioo cc de solution renfermant :

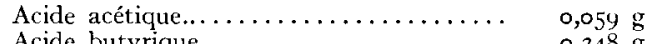

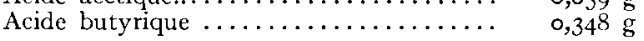

Valeurs trouvées par dosage dans le mélange :

\begin{tabular}{|c|c|c|}
\hline & Acide acétique & Acide butyrique \\
\hline Mini & 0,059 & 0,348 \\
\hline & 0,066 & 0,355 \\
\hline ne ....... & 0,062 & 0,351 \\
\hline Rendement \% & $105,1+1,02$ & $100,0+0.58$ \\
\hline
\end{tabular}

$2^{\circ}$ Mélange de $80 \%$ d'acide acétique et de $20 \%$ d'acide butyrique en solution $\mathrm{N} / 20$.

Prise d'essai : Ioo cc de solution contenant :

Acide acétique....................... $0,238 \mathrm{~g}$

Valeurs trouvées par dosage dans le mélange:

\begin{tabular}{|c|c|c|}
\hline & Acide acétique & Acide butyrique \\
\hline Minima............. & 0,237 & 0,085 \\
\hline Maxima..$\ldots \ldots \ldots \ldots$ & 0,247 & 0,092 \\
\hline Moyenne .............. & $0,24[$ & 0,089 \\
\hline Rendement $\% \ldots \ldots \ldots$ & $101,3+0,35$ & $102,3+0,62$ \\
\hline
\end{tabular}

b) VÉRIFICATION A L'AIDE D'UNE PULPE ADDITIONNÉE DE QUANTITÉS VARIABLES D'ACIDE ACÉTIQUE ET BUTYRIQUE EN ABSENCE ET EN PRÉSENCE D'ACIDE LACTIQUE

Nous avons préparé un extrait aqueux de pulpes sèches que nous avons partagé en trois fractions. La première de ces fractions a été soumise à un dosage d'acides volatils et d'acide lactique, selon la technique IEPPER-FLIEG. La seconde a été additionnée de quantités connues, mais variables, d'acides acétique et butyrique purs, et la troisième, additionnée de ces mêmes quantités d'acides volatils, l'a été également d'une quantité donnée d'acide lactique pur. Quatre déterminations successives ont été effectuées sur chacune des fractions, avec une prise d'essai de $200 \mathrm{cc}$.

I ${ }^{0}$ Dosage sur la fraction de l'extrait aqueux, de pulpe, non additionné d'acides :

$$
\begin{aligned}
& \text { Acide acétique................. } 0,037 \mathrm{~g} \\
& \text { Acide butyrique } \ldots \ldots \ldots \ldots \ldots \ldots \ldots \ldots, 0,002 \mathrm{~g}
\end{aligned}
$$

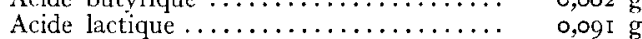

$2^{0}$ Dosage sur une prise d'essai de la fraction d'extrait additionnée d'acides acétique et butyrique en absence d'acide lactique :

Quantités présentes théoriquement dans la prise d'essai. Quantités dosése par la technique LePPer.........

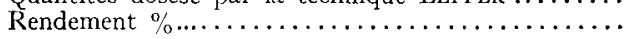

$$
\begin{array}{cc}
\text { Acide acétique } & \text { Acide butyrique } \\
- & - \\
0,440 \mathrm{~g} & 0, \mathrm{I} 46 \mathrm{~g} \\
0,459 \mathrm{~g} & 0, \mathrm{I} 49 \mathrm{~g} \\
104,1 \% \mathrm{I} \% & 102, \mathrm{I} \%
\end{array}
$$


$3^{0}$ Dosage sur une prise d'essai de la fraction d'extrait additionnée d'acides acétique et butyrique en présence d'acide lactique :

Quantités présentes théoriquement dans la prise d'essai .............. Quantités dosées par la technique

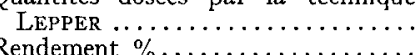

$\begin{array}{ccc}\text { Acide acétique } & \text { Acide butyrique } & \text { Acide lactique } \\ 0,438 \mathrm{~g} & 0,148 \mathrm{~g} & 0,307 \mathrm{~g} \\ 0,449 \mathrm{~g} & 0,152 \mathrm{~g} & - \\ 102,5 \% & 102,7 \% & \end{array}$

Nous avons conclu, d'après ces résultats, que la précision de la méthode était satisfaisante et qu'elle permettait de procéder à une évaluation correcte de quantités d'acides gras volatils apportées par les ingesta de nos animaux d'expérience.

\section{C. - Technique de dosage des lipides dans le lait}

Le choix d'une méthode convenable de dosage des matières grasses dans le lait était pour nous de première importance. Outre la précision demandée à la méthode, celle-ci devait également permettre d'effectuer journellement 25 à 30 déterminations, c'est-à-dire être en même temps d'exécution très rapide.

La technique de Rose-GotTries est évidemment la plus classique et la plus précise des méthodes préconisées, mais elle est excessivement lente et pratiquement inutilisable en dehors d'un laboratoire, ce qui précisément était notre cas.

Aussi avons-nous adopté la méthode de GERBER, qui dérive de celle de BABCock. Sa précision est très voisine de celle de Rose-GotTliteb (I3), tout en étant beaucoup plus rapide que cette dernière et fort pratique en même temps.

Comparant la méthode Rose-GotTlieb et celle de GERBER, ORLAJENSEN (52) constate un écart maximum entre les résultats fournis par ces deux techniques, de $0,4 \%$. VAN DEN BRUG (59) trouve pour 85 échantillons de lait analysés, une teneur moyenne de $3,4 \mathrm{I} 4 \%$ de matière grasse par la méthode de Rose-GotTtuiEb et 3,409 d'après celle de Gerber.

En vérifiant la méthode Gerber par celle de ROSE-GotTtLIEB, nous avons trouvé, sur ro laits de provenance différente, les moyennes que voici:

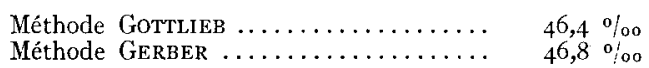

Nous avons cependant relevé quelques écarts entre les dosages effectués en double, dont nous citons les plus caractéristiques :

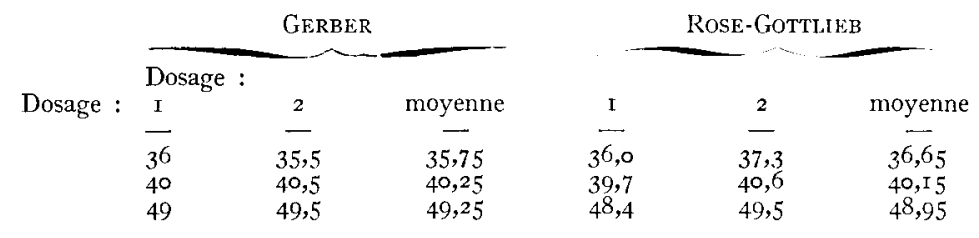


Principe du dosage de la matière grasse par la méthode de GERBER : Le dosage comporte une destruction de l'émulsion caséine-matière grasse dans le lait, par attaque à 1'acide sulfurique. L'élévation de température $\left(70-80^{\circ} \mathrm{C}\right)$ qui s'ensuit favorise la solubilisation de la caséine dans l'excès d'acide. Les globules gras ainsi libérés sont réunis par centrifugation dans la partie graduée du butyromètre de GERBER, dont les divisions correspondent à des grammes de matière grasse rapportés au $\mathrm{kg}$ de lait. L'addition d'une petite quantité de solvant ( $\mathrm{cc}$ d'alcool amylique) facilite la séparation de la matière grasse et son accumulation dans le tube gradué lors de la centrifugation qui doit s'effectuer pendant 5 minutes et être suivie d'un chauffage au bain-marie à $65^{\circ}$ de même durée.

La prise d'essai est de II cc de lait qui sont attaqués par Io ce d'acide sulfurique concentré de densité 1,825 .

\section{CHAPITRE IV}

\section{ETABLISSEMENT DES REGIMES D'EXPERIENCE}

Lors de la constitution des régimes alimentaires de nos vaches d'expérience, nous nous sommes préoccupés :

a) d'adapter la structure physique de la ration et son volume à la physiologie du tube digestif du ruminant, afin de maintenir l'animal dans des conditions de nutrition aussi naturelles que possible.

Nous avons, en conséquence, donné une fixité relative à la partie du régime qui, par nécessité physiologique, doit comporter des aliments grossiers (fourrages secs) apportant une certaine quantité de lest indispensable, et des aliments succulents (racines de betteraves ou ensilage), dont l'action légèrement laxative permet d'entretenir le tube digestif de la vache en lactation dans un bon état de fonctionnement physiologique ;

b) d'adjoindre à cette partie fixe une autre, variable avec les fluctuations des besoins individuels et comportant des aliments concentrés (tourteaux oléagineux; céréales, issues et mélange salin) qui, sous un volume restreint, facilitent à compléter la couverture des dépenses globales de l'organisme, compte tenu des quantités d'énergie et de protides fournies par la partie fixe de la ration.

\section{A. - Normalisation d'un régime de référence}

Notre premier souci, lors du choix des aliments devant composer notre régime de référence, était d'y introduire le moins possible d'acides gras volatils. Fort heureusement, les aliments courants de la vache laitière, nous l'avons signalé dans la première partie de notre travail (voir 
p. I4I), renferment seulement des quantités négligeables de ces substances. Les dosages préliminaires auxquels nous avons soumis les aliments destinés à nos expériences nous ont fait enregistrer des teneurs en acides volatils excessivement faibles, que montrent les résultats ci-après :

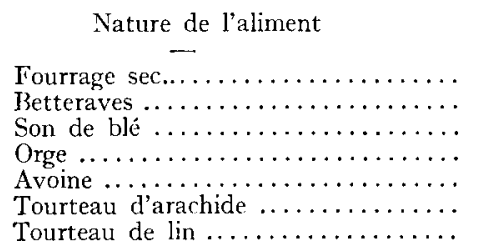

$\begin{array}{cc}\begin{array}{c}\text { grammes } \mathrm{P} \text { Ioo } \\ \text { de matière sèche } \\ \text { Acétique } \\ -\end{array} & \begin{array}{c}\text { Acide } \\ \text { butyrique }\end{array} \\ 0,25 & - \\ 0,88 & - \\ 0,09 & 0,02 \\ 0,09 & - \\ 0,08 & - \\ 0,28 & -0,02 \\ 0,09 & -\end{array}$

\begin{tabular}{cc}
$\begin{array}{c}\text { grammes p. I ooo } \\
\text { d'aliment brut }\end{array}$ \\
\hline $\begin{array}{c}\text { Acide } \\
\text { acétique }\end{array}$ & $\begin{array}{c}\text { Acide } \\
\text { butyrique }\end{array}$ \\
2,25 & - \\
0,95 & - \\
0,78 & $-0,03$ \\
0,77 & - \\
0,70 & - \\
2,56 & - \\
0,80 & -
\end{tabular}

Nous avons pu ainsi limiter I'apport exogène d'acides gras volatils présents dans le régime de référence, à une soixantaine de grammes au maximum.

Nous avons également cherché à réaliser la meilleure association alimentaire possible convenant par ses qualités à 1'activité physiologique de la glande mammaire. De ce point de vue, les fourrages secs, les betteraves, les céréales et issues, les tourteaux de lin et d'arachide, se complètent parfaitement par leurs propriétés physiques, la qualité de leurs protides et leur teneur en phosphore et calcium. L'association de ces divers aliments permet ainsi de constituer un excellent régime pour la vache en lactation (50).

Le régime de référence que nous avons été a menés à normaliser pour l'ensemble de nos recherches, comportait par conséquent une partie fixe identique pour tous les animaux de la même expérience, et une partie variable complémentaire destinée à couvrir le reliquat des besoins individuels qui, comme on le sait, sont fonction du niveau de production de chaque sujet.

\section{I $^{\mathrm{o}}$ Partie fixe du régime}

Cette partie était composée de :

a) betteraves demi-sucrières contenant 9 à I2 \% de matière sèche. Cet aliment est paurre en protides ( 0,8 à $I, 2 \%$ ) dont $20 \%$ seulement sont de nature protéique, le reste se trouvant sous forme d'azote non différencié (bétaïne, asparagine). Il contient également très peu (I à $2 \%$ ) de cellulose et presque pas de lipides, qui sont d'ailleurs inassimilables par le ruminant. Les glucides, par contre, ont une digestibilité très élevée, approchant 95 à $98 \%$.

b) foin de pré ou de luzerne, dont 4 à $7 \mathrm{~kg}$ étaient quotidiennement fournis à chaque animal selon l'importance et la nature de réserves fourragères de l'exploitation où se déroulaient nos essais. 
Le foin est un aliment très cellulosique (30 à $40 \%$ ) qui apporte le lest nécessaire. Il est riche en matière sèche $(85$ à $87 \%$ ) et renferme 4 à $6 \%$ de matières protéiques digestibles de valeur biologique élevée, parmi lesquelles figure une certaine quantité de lysine et de tryptophane. Il contient également des acides gras supérieurs, en particulier des acides oléique et linoléique.

\section{$2^{\circ}$ Partie variable complémentaire}

La règle la plus difficile à satisfaire dans un plan de rationnement destiné à des vaches laitières, est celle concernant la concordance simultanée de 1'équilibre énergétique et protéique recherché pour chaque sujet. Nous avons résolu la difficulté en fabriquant deux mélanges concentrés complémentaires isodynames, dissemblables par leur teneur en protéine digestible, mais préparés à partir des mêmes éléments de base associés en proportions différentes. Ainsi, le mélange riche en protéines (A) était à prédominance de tourteaux, tandis que dans le concentré pauvre en protéines (B), les céréales l'emportaient. Des quantités adéquates des mélanges $\mathrm{A}$ et $\mathrm{B}$, calculées d'après les besoins individuels des vaches, permettaient d'atteindre ainsi l'équilibre nutritif recherché.

Les deux aliments composés en question comportaient des tourteaux de lin et d'arachide, du son de blé, de l'avoine et de l'orge. Il a été incorporé à ces concentrés $3 \%$ de la formule saline 4-3-2-I recommandée par LEROY (44), comprenant : phosphate tricalcique : 4 parties $-\mathrm{NaCl}$ : 3 parties - $\mathrm{CaCO}_{3}: 2$ parties - et $\mathrm{SO}_{4} \mathrm{Mg}:$ I partie.

Ce mélange salin avait pour but de parer, dans la mesure du possible, à des carences phospho-calciques et sodiques chez nos animaux d'expérience.

\section{B. - Régimes d'expérience enrichis en acides gras volatils}

Lors de nos essais d'enrichissement en acides gras volatils des régimes d'expérience, nous nous sommes efforcé de modifier le moins possible l'association alimentaire du régime de référence, afin d'éviter que des changements qualitatifs autres que ceux résultant de l'introduction de ces substances, puissent influer sur nos résultats.

\section{ro Régime expérimental à base d'acides gras volatils formés à l'état naturel}

Toutes les autres conditions étant égales par ailleurs, la seule modification apportée au régime de référence a consisté dans une substitution aux betteraves qui y figurent, poids pour poids, d'un ensilage mixte de 
pulpes et de collets de betteraves sucrières ; ce dernier renferme effectivement des acides acétique et butyrique, naturellement formés dans la masse au cours d'une fermentation non dirigée.

Nous avons préféré ce type d'ensilage, parce qu'il se rapproche le plus de la betterave du régime témoin, par ses origines botaniques, par la nature, la qualité et le taux de ses principes nutritifs.

L'aliment était distribué journellement, au cours des périodes expérimentales, à raison de $45 \mathrm{~kg}$ par vache. La légère différence en énergie et en matières protéiques digestibles résultant du remplacement des betteraves par de l'ensilage, était prise en considération pour le calcul de la couverture des besoins individuels des animaux en expérience.

Des échantillons d'ensilage, prélevés deux fois par semaine au cours de l'expérience et transportés immédiatement au laboratoire, étaient soumis aussitôt aux dosages des acides gras volatils et de l'acide lactique selon la technique de LEPPER-FLIEG. Les résultats moyens hebdomadaires de ces déterminations, exécutées en double, figurent ci-contre :

\section{Essai I (hiver I949)}

Moyennes hebdomadaires en $\mathrm{g} \%$ de produit brut acide acétique acide butyrique acide lactique

I ${ }^{\circ}$ Période expérimentale $b$ :

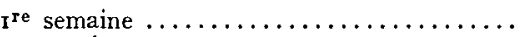

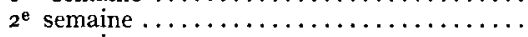

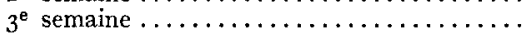

Teneur moyenne ...................

Quantités totales ingérées journellement par vache.

\begin{tabular}{l}
12,46 \\
10,02 \\
10,80 \\
\hline 11,09 \\
\hline 499
\end{tabular}

\begin{tabular}{lr}
7,74 & 3,40 \\
7,96 & $\mathrm{I}, 59$ \\
7,68 & $\mathrm{I}, 65$ \\
\hline 7,79 & $2,2 \mathrm{I}$ \\
\hline $35 \mathrm{I}$ & \\
\hline
\end{tabular}

$2^{0}$ Période expérimentale $c$ :

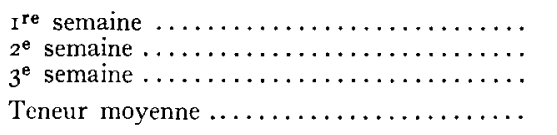

Quantités totales ingérées journellement par vache.

$\begin{array}{r}8,91 \\ 12,41 \\ 9,46 \\ \hline 10,26 \\ \hline 464\end{array}$

\begin{tabular}{ll}
9,57 & 2,74 \\
7,44 & 3,16 \\
5,32 & 5,77 \\
\hline 7,45 & 3,89 \\
\hline 333 &
\end{tabular}

$$
\text { Essai II (hiver I950) }
$$

10 Période expérimentale $b$ :

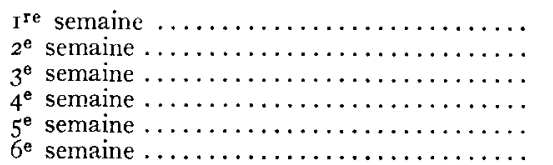

Teneur moyenne ...................

Quantités totales ingérées journellement par vache.

$$
\begin{array}{r}
10,00 \\
8,40 \\
6,70 \\
10,17 \\
9,80 \\
9,70 \\
\hline 9,13 \\
\hline 393
\end{array}
$$

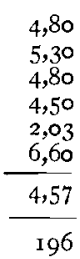




\section{$2^{\circ}$ Régime expérimental à base d'acides gras volatils d'origine synthétique}

Il renfermait le régime de référence tel que et des acides gras volatils de synthèse, incorporés dans ce dernier sous forme d'acétate et de butyrate de calcium. Ces produits ont été préparés à notre intention lors de chaque essai, en une seule fois, par un laboratoire privé.

Le recours à des sels de sodium de ces substances, plus solubles que ceux de calcium, aurait certes été préférable, mais nous craignions qu'ingérés sous cette forme, les produits ne perturbent l'équilibre de la réserve alcaline du sang. Aussi y avons-nous renoncé.

D'après l'analyse effectuée par nos soins, les sels de calcium des acides volatils utilisés au cours des essais, avaient la composition que voici :

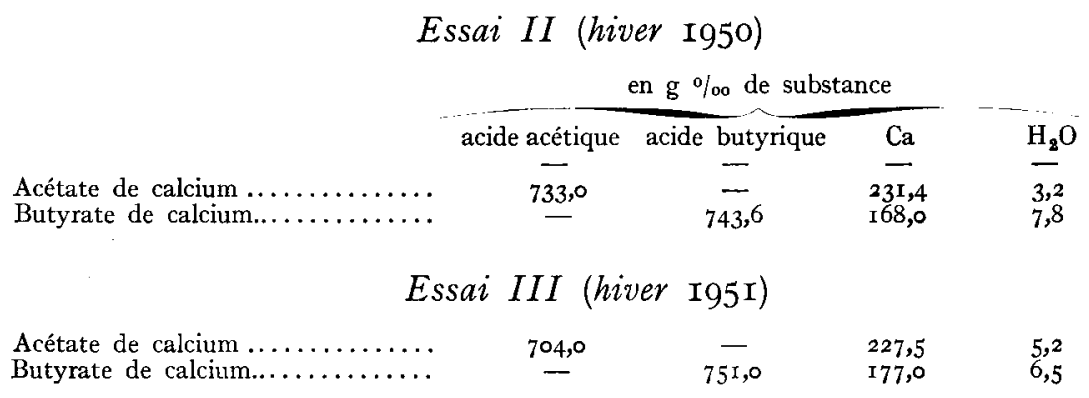

CHAPITRE V

\section{MESURES EXPERIMENTALES ET METHODES D'INTERPRETATION DE RESULTATS D'EXPERIENCE}

\section{A. - Evaluation des ingesta individuels}

Des stocks suffisants d'aliments, constitués pour chacun des essais, nous permettaient d'éviter des variations trop grandes dans l'origine et la composition chimique des ingesta, au cours de périodes successives de la même expérience.

Une analyse chimique exécutée fréquemment pour chaque aliment, servait à l'estimation de la valeur nutritive de la ration alimentaire.

En raison de leur grande altérabilité, les betteraves et les ensilages étaient analysés deux fois par semaine. Pour éviter également des erreurs qui auraient pu résulter de la grande hétérogénéité des réserves de foin, des quantités adéquates de cet aliment étaient prélevées journellement 
et gardées dans la même enceinte que celles-ci. Elles servaient à l'issue de chaque période, à la préparation d'un échantillon moyen périodique de 2,5-3 kg destiné à l'analyse chimique. Les tourteatix, les céréales et le son provenant des stocks et entrant dans la composition de mélanges concentrés complémentaires étaient analysés lors de la préparation de ces derniers.

Chaque vache en expérience recevait journellement le poids d'aliments qui lui revenait d'après les normes. Le calcul de rations était effectué deux fois par semaine, compte tenu de l'évolution de la sécrétion lactée et du poids vif de 1'animal.

Les rations journalières étaient distribuées en trois fois : un premier repas, réparti à la traite du matin, comportait une demi-ration de betteraves et la moitié du foin ; un second repas, constitué par la totalité de la ration de mélange concentré, était distribué peu avant le début de la traite du soir ; le dernier repas, enfin, comprenait les reliquats de betteraves et de foin et était mis à la disposition des animaux aussitôt cette traite achevée.

Les sels de calcium d'acides gras volatils étaient incorporés dans 1a ration individuelle des betteraves, au moment de la distribution et mélangés intimement à la masse.

\section{B. - Mesures des sécrétions lactées et lipidiques individuelles}

Les vaches étaient traites régulièrement chaque jour, le matin et le soir. Les très fortes laitières subissaient cependant une traite supplémentaire, vers 1'heure de midi.

A chaque traite, le lait individuel était récolté dans un seau taré, pesé à l'aide d'une balance sensible à $25 \mathrm{~g}$ et le poids net observé inscrit sur un carnet d'étable, où étaient enregistrés les animaux. Après homogénéisation du contenu de la traite, on procédait au prélèvement d'un échantillon de lait destiné au dosage de la matière grasse, au moyen d'une pipette de $20 \mathrm{cc}$ graduée au I/IO.

La détermination de la teneur des laits individuels en matière grasse s'effectuait par la méthode de GERBER, sur un échantillon moyen journalier, composé proportionnellement aux quantités de lait sécrétées à chaque traite par la vache.

Les quantités totales de matières grasses élaborées journellement par l'animal étaient obtenues par calcul, en multipliant la quantité de lait récoltée dans la même journée par sa richesse lipidique correspondante.

$\mathrm{I}_{\text {a }}$ production journalière de lait était en outre transformée à l'aide de la formule de GaINEs, en lait corrigé à $4 \%$ de matière grasse, pour faciliter le calcul des besoins alimentaires de chaque sujet. 


\section{C. - Mesure des variations du poids corporel des animaux}

Les sujets étaient pesés individuellement, à l'entrée et à l'issue de chaque période, pendant trois jours consécutifs. Il était en outre procédé à une pesée hebdomadaire durant la période même. Ces diverses opérations étaient exécutées à la même heure et dans des conditions identiques, afin de les rendre aussi précises que possible.

\section{D. - Méthodes d'interprétation des résultats}

L'interprétation statistique permet de prouver, avec une grande rigueur scientifique, la validité des résultats d'une expérience. La statistique cependant, obéit à la loi des grands nombres, qu'une étude de nutrition sur la vache laitière peut rarement satisfaire.

Les conditions particulières dans lesquelles nous avons été amenés à travailler ne nous ont, à notre grand regret, pas permis d'expérimenter sur un grand nombre d'animaux à la fois. En l'occurrence, il importe donc, croyons-nous, d'insister davantage sur l'évidence physiologique des observations que sur leur signification statistique, sous la réserve toutefois que les méthodes expérimentales adoptées ne soient pas critiquables en elles-mêmes.

Nous avons toutefois tenu à soumettre nos résultats à quelques tests statistiques, afin de nous assurer que les observations recueillies ne procèdent pas seulement du hasard et d'évaluer les chances qu'elles ont d'approcher la vérité mathématique. Nous signalons plus loin les formules classiques qui permettent d'effectuer les tests en question, ainsi que leur référence $(60)$.

\section{Io Test d'homogénéité}

L'épreuve statistique des résultats comporte les calculs de la variance commune de deux moyennes, selon la formule:

$$
\mathrm{S}\left(\overline{\mathrm{X}}_{1}-\overline{\mathrm{X}}_{2}\right)=\frac{\mathrm{\Xi}\left(\mathrm{X}_{1}-\overline{\mathrm{X}}_{1}\right)+\mathrm{Y}\left(\mathrm{X}_{2}-\overline{\mathrm{X}}_{2}\right)}{\mathrm{N}_{1}+\mathrm{N}_{2}-2}
$$

et le test du $t$, de STUDENT Fisher, d'après la formule :

$$
t=\frac{\mathrm{N}}{2} \cdot \frac{\overline{\mathrm{X}}_{1}-\overline{\mathrm{X}}_{2}}{\mathrm{~S}\left(\overline{\mathrm{X}}_{1}-\overline{\mathrm{X}}_{2}\right)}
$$

Ce test nous a servi à vérifier l'homogénéité des groupes d'animaux formés pour l'expérience, et à nous convaincre en même temps, que les échantillons ainsi constitués faisaient partie d'une même population.

Il nous a également permis d'apprécier la signification statistique 
des écarts constatés entre deux séries d'observations. Rappelons, à cet effet, que toute différence dont la valeur du $t$ dépasse 2 est considérée comme significative, au seuil de probabilité de $5 \%$.

$2^{\circ}$ Calcul de l'écart type de la moyenne

L'opération nécessite :

$$
\begin{aligned}
& \text { une détermination de la variance } \quad \sigma^{2}=\frac{\Sigma(\mathrm{X}-\overline{\mathrm{X}})^{2}}{\mathrm{~N}-\mathrm{I}}
\end{aligned}
$$

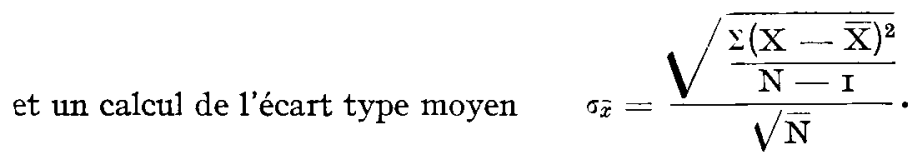

\section{$3^{\circ}$ Test d'ajustements linéaire et exponentiel.}

Le tracé de la ligne de régression théorique des sécrétions lactée et lipidique observées au cours de l'expérience, a été soumis à un test d'ajustement. Ceci nous a donné la possibilité de choisir parmi les deux régressions, la linéaire et l'exponentielle, celle dont nos observations réelles s'approchaient le plus.

Ce test implique 1'adoption de celui des modes d'ajustement, dont la somme des carrés des écarts existant entre les données théoriques $\left(\mathrm{Y}_{t}\right)$ déduites de la régression et les valeurs réellement observées ( $\mathrm{Yr}$ ), est la plus faible. Précisons que l'expression

$$
\Sigma\left(Y_{t}-Y_{r}\right)^{2}
$$

permet de calculer la somme en question.

Nos calculs nous ont prouvé que, dans la plupart des cas, la régression exponentielle rajustait mieux que la linéaire nos données réelles de la production laitière. Le tracé de nos courbes théoriques a donc été fait selon cette méthode, dont le choix se trouve également justifié par une très récente étude systématique (4I).

A notre connaissance, le type d'ajustement qu'il convient d'adopter pour la sécrétion lipidique n'a pas encore été étudié pour l'instant. Nous avons donc préféré y appliquer, comme KELLNER, la régression linéaire.

\section{$4^{\mathbf{0}}$ Test de linéarité}

Pour nous assurer que les régressions exponentielles de la sécrétion lactée et celles linéaires de la sécrétion lipidique représentent effective- 
ment nos données réellement observées, nous avons eu recours à deux formules de calcul de variance :
a) Variance interclasses
$\mathrm{S}^{2}{ }_{\mathrm{D}}=\frac{\mathrm{\Sigma}(\mathrm{Y} i-\mathrm{Y} i)^{2} \cdot n}{\mathrm{~K}-2}$
b) Variance intraclasses
$\mathrm{S}_{\mathrm{I}}^{2}=\frac{\left(\mathrm{Y} i-\widehat{\mathrm{Y}}_{i}\right) \cdot \mathrm{K}}{\mathrm{N}-\mathrm{K}}$.

Pour que la linéarité soit acceptable, la variance $S^{2}{ }_{p}$ doit être égale, inférieure ou non significativement plus grande que celle de $\mathrm{S}^{2}{ }_{\mathrm{I}}$.

\section{(à suivre)}

\section{BIBLIOGRAPHIE}

(I) Aliman (R. T.), Hamilton (T. S.). - Nutrit. Defic, in Livest. F. A. O. Agric. Stud., I948, 5.

(2) Armsby (H. P.), Fries (J. A.), Braman (W. W.). - Proceed. Nat. Sci., I9I8, 4, I.

(3) Armsby (H. P.). - The Nutrition of Farm Animals (Mc Mrldan N. Y.), I9I7.

(4) Bartiett (S.). Blaxter (K.). - J. Agric. Sc., I947, 37, 32.

(5) Blackwood (J. H.). Stiri. (J. D.). - Bioch. J., I932, 26, 357.

(6) BotTini (E.). - Ann. St. Chim. Agr. Torino, I938-40, 14, 4I.

(7) BREIREM (K.). - XII Congrès International de Laiterie, I949, 1, 30.

(8) Brody (S.). - Bioenergetics and Growth (Reinhold N. Y.), I944.

(9) Brody (S.), Cunningham (R.). - Miss. Agr. Exp. Stat. Bull., I936, 238, 52 et 244,56 .

(Io) Buschmann (A.). - Landw. Vers. Stat., I923, 101, r.

(iI) Cole (H. H.), Mead (S. W.), Kleiber (M.). - Califo. Agr. Exp. Stat. Bull. I942, 662, 22.

(I2) Delage (J.), Françors (A.). - Comm. Ve Congrès Int. Zoot., Paris, I949, p. 5 .

(13) EisSDEN (G. D.), WALKER (C. H.). - Richmond Dairy Chemistry, I942, p. $26 \mathrm{I}-266$.

(I4) Evans (H. M.), Burr (G. O.). - J. Biol. Chem., I928, 76, 263.

(I5) Ferrando (R.). - C. R. Acad. Agric., Paris, I950, 36, 354.

(I6) Forbes (E. B.). - A gric. Exp. Stat. Bull., I935, 319, I52.

(I7) Forbes (E. B.), Kriss (M.). - Proc. Soc. Anim. Prod., I931, p. II3I.

(I8) Forbes (E. B.), KRISS (M.). - J. Nutrit., I932, 5, I83.

(I9) Forbes (E. B.). - a) Ohio Stat. Res. Bull. I92 I, 34\%, 99. - b) J. Biol. Chem., I922, 52, $28 \mathrm{I}$.

(20) Frens (H. M.). - Land Tidschr., I942, 84, 40.

(2I) Fuchs (P.). - Tierernähr. u. Futtermitt., I943, 6, 89.

(22) Gaines (W. L.). - Ill. A gr. Exp. Stat. Bull., I928, 308, 40 I.

(23) GNEIST (K.). - Tierernähr, I932, 4, I85.

(24) Halnan (M. A.). - J. Dairy Res., I929, 1, 3.

(25) Hansen (A. E.). - Futterung der Haustiere, 1926.

(26) Hart (E. B.), Humphrey (G. C.). - Cités par no 49.

(27) Hastings (W. H.). - J. Dairy Sci., I944, 27, Ior5.

(28) Henke et Goo. - Cités par no 49.

(29) Hilıs (J. L.). - Verm. A gr. Exp. Stat. Bull., I922, 225, 3.

(30) Honcamp (E.), Koudela (S.). - Bioch. Zeits., I923, 143, i II.

(3I) HoRn (W.). - Bied. Zentralbl. B. Tierernähr., I934, 6, 44I.

(32) JORDAN. - Cité par $n^{\circ} 3$. 
(33) Kax (H. D.). - Médecine et Biologie, I947, 6.

(34) Kellner (O.). - Ernähr. Landw. Nutztiere, Berlin, Igio, p. 533.

(35) KELLNER (O.). - Communication au Ve Congrès International pour 1'Industrie laitière, IgII.

(36) KELlNeR (O.). - Landw. vers. Stat., I900, 53, 4 I5.

(37) KIEFERLE, Cité par $n^{\circ} 49$.

(38) Kirsch (W.), JanTzON (H.). - Zeit. f. Zücht. u. Tiernähr., I933, 28, 45I.

(39) LEPPER (W.). - Tierernähr. u. Futtermitt, I938, 1, I87.

(40) LEPPER (W.). - Landw. Vers. Stat., I933, 11\%, II3.

(4I) Leroy (A. M.), Delage (J.), Poly (J.). - Ann. Zoot., I952 (à paraître).

(42) Leroy (A. M.). - C. R. Acad. Agric., Paris, I937, 23, 67.

(43) Leroy (A. M.), Zeiter (S. Z.). - Ann. Agronomiques, I948, 18, 78.

(44) LEROY (A. M.). - Elevage rationnel des animaux domestiques (Hachette), Paris, I943.

(45) MC EILROY (I. W.), Goss (H.). - J. Nutrit., I94I, 21, I63 et 405.

(46) MEEH. - Zeitschr. f. Biol., I879, 15, 425.

(47) Mitcheli (P. H.). - Biochem. of Amino-Acids, I929.

(48) MollgaARd (D. H.). - Grïnd. Ermähr. Phys. d. Haust., Berlin, I93I, p. 3IO.

(49) Morris (S.). - - Nutrition et Lactation, Act. Sci. et Ind., I938.

(50) Morrison (F. B.). - Feeds et Feeding (Ithaca), I936.

(5I) Osborne (T. B.), Mendel, (L. B.). - J. Biol. Chem., I9I2, 12, 473.

(52) ORLA-JENSEN (S.). - Le Lait, I923, 3, I76.

(53) Owen (E. C.), SMi'T (J. A. B.), WRight (N. C.). - Bioch. J., I943, 37, 44.

(54) Petersen (W. E.). - Proc. Soc. Exp. Biol., I932, 3o.

(55) Savage (E. S.). - Corn. Univ. A gric. Exp. Stat. Bull., I933, 578.

(56) Savage (E. S.). - Corn. Univ. Agr. Exp. Stat. Bull., 1935, 622.

(57) Smith (R. H.), Rice (J. B.). - Guernesey Breed. J., I934, 46, I76.

(58) Terroine (E. F.). - Le métabolisme de l'azote, Paris, I933.

(59) VAN DEN BRUG. - Net. Off. Org. Zuiwelbond., r929, I9.

(60) Vessereau (A.). - Méthodes statistiques en Biologie et en Agronomie (Baillère), Paris, I948.

(6i) Virtanen (A. I.), Pulkiti. - J. Am. Chem. Soc., I928, 50, 3I.

(62) Watson (C. J.). Davidson (W. A.), Kennedy (J. W). - J. Agric. Sci., I949, 29, I85.

(63) Watson (C. J.). - J. Agric. Sci., I936, 26, 337.

(64) Wiegner (G.), Magasanik (J.). - a) Lebensmitt. u. Hygienne, agig, 10, I56. - b) Biederm. Zentralbl., I922, 51, I40.

(65) Winegar (A. H.), Pearson (P. B.), Schmidt (H.). - Sci. (N. Y.), I940, 91, 508 . 